\title{
PI3K/AKT signaling determines a dynamic switch between distinct KSRP functions favoring skeletal myogenesis
}



Skeletal myogenesis is orchestrated by distinct regulatory signaling pathways, including PI3K/AKT, that ultimately control muscle gene expression. Recently discovered myogenic micro-RNAs (miRNAs) are deeply implicated in muscle biology. Processing of miRNAs from their primary transcripts is emerging as a major step in the control of miRNA levels and might be well suited to be regulated by extracellular signals. Here we report that the RNA binding protein KSRP is required for the correct processing of primary myogenic miRNAs upon PI3K/AKT activation in myoblasts $\mathrm{C} 2 \mathrm{C} 12$ and in the course of injury-induced muscle regeneration, as revealed by Ksrp knock-out mice analysis. PI3K/AKT activation regulates in opposite ways two distinct KSRP functions inhibiting its ability to promote decay of myogenin mRNA and activating its ability to favor maturation of myogenic miRNAs. This dynamic regulatory switch eventually contributes to the activation of the myogenic program.

Cell Death and Differentiation (2012) 19, 478-487; doi:10.1038/cdd.2011.117; published online 2 September 2011

Myogenic differentiation is the end point of a cascade of events that remove myoblasts from the cell cycle and ultimately determine the characteristic pattern of skeletal muscle gene expression and the formation of multinucleated myotubes. ${ }^{1}$ Once differentiated, adult muscles retain the capacity to regenerate in response to exercise or injury by activation and proliferation of satellite cells. ${ }^{2,3}$ Skeletal myogenesis is orchestrated by distinct regulatory signaling pathways, including phosphatidylinositol-3-kinase (PI3K)/ AKT, that ultimately change tissue-specific gene expression programs during muscle cell differentiation and tissue reconstruction after trauma. ${ }^{4}$ In the recent few years, extensive analysis of the composition of the transcriptome assembled on the chromatin of myogenic loci has been performed (reviewed in Guasconi and Puri ${ }^{5}$ ), although knowledge on the mechanisms that post-transcriptionally regulate muscle differentiation and regeneration is more limited. ${ }^{6-9}$

Recently, genetic, and biological evidence of an essential role of microRNAs (miRNAs) in muscle development and function has been accumulating and a group of miRNAs, highly enriched in skeletal muscle (referred to as myomiRs), has been identified (reviewed in Williams et al., ${ }^{10}$ and Güller and Russell ${ }^{11}$ ). MyomiRs influence multiple aspects of muscle development and function through their regulation of key genes controlling myogenesis. ${ }^{12-14}$ Deregulated expression of some myomiRs results in pathological conditions. ${ }^{15-17}$
miRNA encoding genes are transcribed as primary miRNA transcripts (pri-miRNAs) by RNA polymerase II. The mechanism by which pri-miRNAs are processed first to stem-loop structured miRNA precursors (pre-miRNAs) and then to mature miRNAs involves two sequential endonucleolytic cleavages operated by multiprotein complexes including the RNase III enzymes Drosha, in the nucleus, and Dicer, in the cytoplasm (reviewed in Krol et al. ${ }^{18}$ ). Mature miRNAs, in the context of the RNA-induced silencing complex (RISC), are able to silence gene expression by binding to partially complementary target sites predominantly located in the $3^{\prime}$ untranslated region ( $3^{\prime}$ UTR) of target messenger RNAs (mRNAs). ${ }^{18}$ This results either in the repression of translation or in the deadenylation and destabilization of the target mRNA (reviewed in Fabian et al. ${ }^{19}$ ).

In the last few years, the relevance of post-transcriptional control of miRNA biogenesis has been discovered (see Newman and Hammond ${ }^{20}$ for a recent review) and, notably, miRNA processing defects result in pathological conditions including cancer. ${ }^{21,22}$ Inactivation of Dicer in muscles results in perinatal lethality due to skeletal muscle hypoplasia, thus highlighting the essential role of the miRNA processing machinery in muscle homeostasis. ${ }^{23}$ Recently, Paroo et al., ${ }^{24}$ reported that MAPK Erk phosphorylates TRBP, a protein factor belonging to the core Dicer complex, and affects miRNA maturation. This observation points to the relevance of

${ }^{1}$ Gene Expression Regulation Laboratory, Istituto Nazionale per la Ricerca sul Cancro, 16132 Genova, Italy; ${ }^{2}$ Department of Biochemistry \& Molecular Genetics, University of Alabama at Birmingham, Birmingham, AL, USA; ${ }^{3}$ University of Genova, 16132 Genova, Italy; ${ }^{4}$ RNA Technology Laboratory, Centro di Biotecnologie Avanzate (CBA), 16132 Genova, Italy and ${ }^{5}$ Howard Hughes Medical Institute, Department and School of Medicine, University of California, San Diego, CA, USA *Corresponding authors: P Briata or R Gherzi, Gene Expression Regulation Laboratory, Istituto Nazionale per la Ricerca sul Cancro, Largo R. Benzi, 10, 16132 Genova, Italy. Tel: + 010573 7540; Fax: + 010573 7405; E-mail: paola.briata@istge.it (P Briata); rgherzi@ucsd.edu (R Gherzi)

${ }^{6}$ Current address: INSERM, U895, Centre Méditerranéen de Médecine Moléculaire (C3M), Control of Gene Expression Laboratory, 06204 Nice, France Keywords: myogenesis; mRNA degradation; microRNA maturation; RNA-binding protein

Abbreviations: PI3K, phosphatidylinositol-3-kinase; miRNAs, microRNAs; pri-miRNAs, primary miRNA transcripts; pre-miRNAs, miRNA precursors; RISC, RNA-induced silencing complex; 3'UTR, 3' untranslated region; mRNAs, messenger RNAs; LY, LY294002; myr-AKT2, myristoilated constitutively active form of Akt2; DM, differentiation medium; GM, growth medium; myr-AKT2/GM, myr-AKT2 expressing C2C12 cells cultured in GM; WT, wild-type; RIP, ribonucleoprotein complexes immunoprecipitation; DRB, 5,6-dichloro-1- $\beta$-D-ribofuranosylbenzimidazole, 5,6-dichlorobenzimidazole riboside

Received 18.5.11; revised 18.7.11; accepted 28.7.11; Edited by M Deshmukh; published online 02.9.11 
post-translational modifications of factors involved in miRNA biogenesis in the modulation of miRNA expression and function. However, regulation of a general molecular machine, such as the Dicer complex, does not explain how different signaling pathways can regulate maturation of different subsets of miRNAs in specialized cellular environments.

It has been recently shown that different regulatory proteins, acting as either activators or repressors of Drosha and Dicer machineries, influence the processing of select miRNAs (reviewed in Trabucchi et al. ${ }^{25}$ ). Among them, the single-strand RNA binding protein KSRP (reviewed in Briata et $a l^{26}$ ) interacts with the terminal loop of select miRNA precursors and promotes their maturation in cultured cells. ${ }^{27-29}$ Importantly, KSRP has been shown to regulate gene expression also promoting the decay of inherently labile mRNAs in several cellular contexts including C2C12 myoblasts. ${ }^{26} \mathrm{KSRP}$ distinct functions are regulated by different protein kinases, including MAPK p38, PI3K/AKT and ATM. ${ }^{8,29,30}$ An unresolved issue is whether a specific signaling pathway regulates only a single KSRP function (i.e. affecting either mRNA decay or miRNA maturation) or is able to induce a dynamic change between KSRP functions in order to produce relevant phenotypes in a specific cellular context.

Here, we report that activation of the PI3K/AKT signaling is responsible for myomiR maturation during $\mathrm{C} 2 \mathrm{C} 12$ myoblasts differentiation with KSRP being required for this function. Importantly, myomiR maturation in the course of injuryinduced muscle regeneration is impaired in Ksrp knock-out mice. PI3K/AKT signaling activation that occurs during myogenesis determines a switch between two distinct KSRP functions, inhibiting its ability to promote the decay of myogenin mRNA while activating its ability to favor maturation of myogenic miRNAs.

\section{Results}

PI3K/AKT signaling induces myomiR maturation in C2C12 myoblasts. In order to assess the contribution of the PI3K/AKT signaling pathway to myomiR induction during differentiation of $\mathrm{C} 2 \mathrm{C} 12$ myoblasts, we continuously exposed cells to PI3K inhibitor LY294002 (LY) throughout the transition from myoblasts to myotubes induced by differentiation medium (DM). LY was able to impair DMinduced expression of myogenic differentiation markers (Supplementary Figure S1a, and Serra et al. ${ }^{31}$ ) and to strongly inhibit the expression of miR-1a, miR-133b and miR206 (members of the myomiR family ${ }^{10,11}$ ) induced by DM (Figure 1a). Importantly, the comparative analysis of primary and mature miRNAs revealed that LY specifically impairs myomiR maturation (Figures $1 a$ and $b$ ). AKT kinases are the major downsteam effectors of $\mathrm{PI} \mathrm{K}^{32}$ As the relative contribution of the two AKT isoforms expressed in C2C12 cells (Akt1 and Akt2) to differentiation is still debated, ${ }^{33}$ we downregulated either isoform before exposure to DM (Supplementary Figure S1b) and obtained inhibition of the expression of myogenic differentiation markers (Supplementary Figure S1c, and Serra et al. ${ }^{31}$ ). Silencing experiments demonstrated that both Akt1 and Akt2 are required for $\mathrm{DM}$-induced maturation of miR-1a, miR-133b and miR-206 (compare the expression of mature and primary miRNA in Figures $1 c$ and d). In agreement with a role of AKT in myomiR maturation, expression of a membrane-bound myristoilated and constitutively active form of Akt2 (myr$\mathrm{AKT}^{30}$ ) strongly enhanced the expression of myomiRs in $\mathrm{C} 2 \mathrm{C} 12$ cells cultured in DM without affecting the expression of the corresponding pri-miRNAs (Figures $1 \mathrm{e}$ and $\mathrm{f}$ ).

PI3K/AKT could cross talk with other signaling pathways activated during DM-induced myoblast differentiation and this could influence myomiR maturation. In order to minimize this potential interference, we activated PI3K/AKT signaling in exponentially growing cells (cultured in growth medium, GM). Indeed, the expression of myr-AKT2 in C2C12 cells cultured in GM (myr-AKT2/GM)-induced the expression of differentiation markers (Supplementary Figure S2a). To obtain a comprehensive picture of the miRNome changes induced by myr-AKT2/GM in C2C12 myoblasts, we performed miRNA microarray analysis and found 37 miRNAs significantly regulated (Figure 2a). As our aim was to investigate the contribution of PI3K/AKT activation in the context of physiological C2C12 myoblast differentiation, we focused exclusively on miRNAs whose expression was similarly affected by myr-AKT2/GM and by DM (Figure 2a and Supplementary Figure S2b). miRNA array results were validated (by both quantitative RT-PCR (qPCR) analysis and RNase protection assays), with the exception of miR-21, whose expression was not affected by myr-AKT2/GM (Figures $2 b$ and c). Importantly, AKT activation enhanced miRNA expression inducing their maturation without significantly affecting the corresponding primary miRNA levels with the only exception of miR-351 whose primary transcript levels were significantly upregulated (Figure 2b).

Insulin and IGF-I are known as physiological activators of $\mathrm{PI} 3 \mathrm{~K} / \mathrm{AKT}$ signaling in different cell types, including $\mathrm{C} 2 \mathrm{C} 12$ myoblasts. ${ }^{34}$ Treatment of serum-starved C2C12 cells with insulin $\left(10^{-6} \mathrm{M}\right)$ was able to induce muscle differentiation markers (Supplementary Figure $2 \mathrm{c}$ ) and to enhance the expression of mature miR-1a, 133b, 206, 378, and 532-5p without increasing the levels of their primary transcripts (Figure 2d and Supplementary Figure 2d).

Connexin 43 is one of the few validated targets of miR-1a and miR-206 ${ }^{13,35}$ and, consistent with the increased expression of these myomiRs, we observed a similar downregulation of connexin 43 protein levels in $\mathrm{C} 2 \mathrm{C} 12$ myoblasts upon either $\mathrm{PISK} / \mathrm{AKT}$ signaling activation or culture in DM (Figure 2e).

Altogether these data demonstrate that either physiological or forced activation of PI3K/AKT signaling in C2C12 myoblasts induces the expression of a group of myogenic miRNAs activating their maturation.

KSRP is required for AKT-induced myomiR maturation. We reported that KSRP is a target of PI3K/AKT signaling in different cell lines, and that phosphorylation by AKT1 and 2 causes an impairment of its decay-promoting function on a variety of inherently labile mRNAs. ${ }^{26}$ We investigated whether KSRP function is required for PI3K/AKT-induced maturation of myomiRs in C2C12 myoblasts and whether KSRP phosphorylation by AKT affects its ability to promote miRNA precursor maturation. 
a

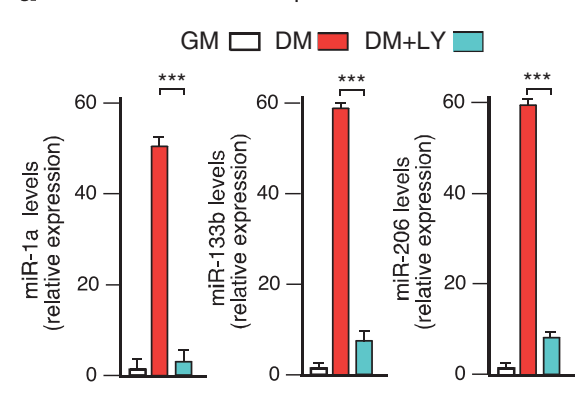

C

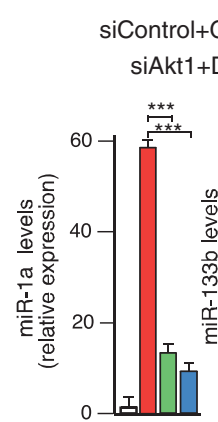

qPCR

I+DM e

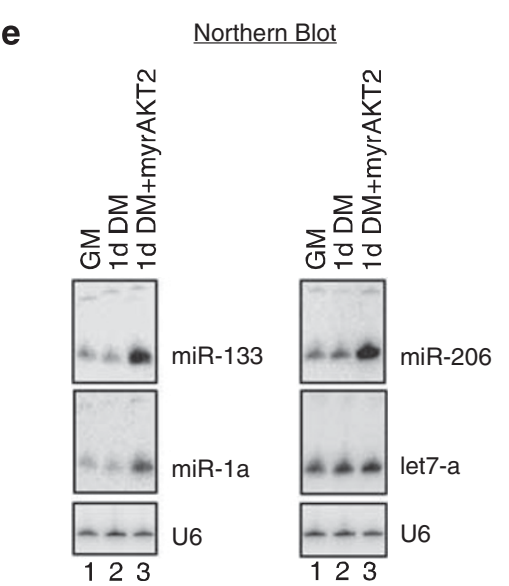

b

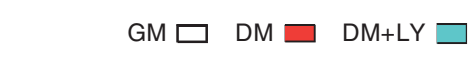

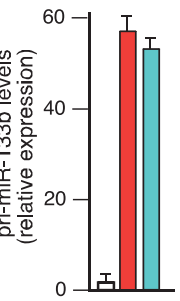

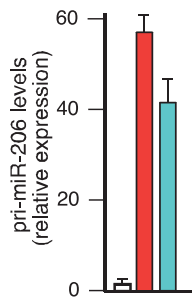

d

qPCR
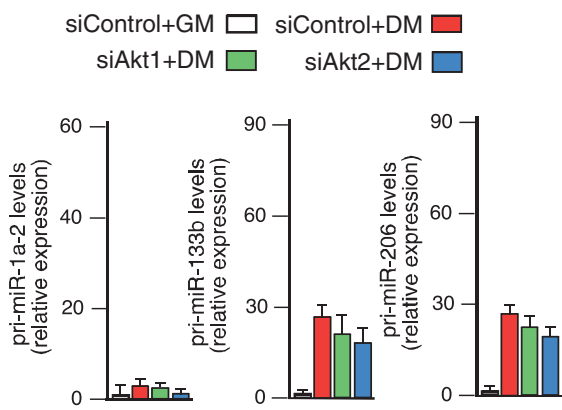

f

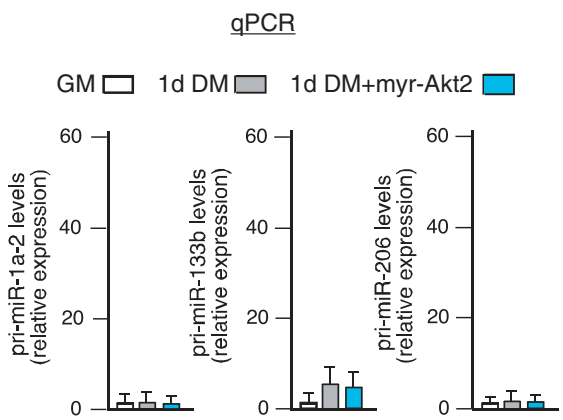

Figure 1 PI3K/AKT signaling controls maturation of myogenic miRNAs in differentiating C2C12 myoblasts. (a and $\mathbf{b}$ ) Quantitative RT-PCR (qPCR) analysis of miR-1a, miR-133b, miR-206 (a), and their respective primary transcripts (b) in C2C12 cells cultured either in growth medium (GM) or differentiation medium (DM) for 2 days in the presence of either DMSO (the solvent of LY294002 (LY), labeled as DM), or LY (25 $\mu \mathrm{M}$, labeled as DM $+\mathrm{LY})$. The values shown are averages ( \pm S.E.M.) of three independent experiments performed in triplicate. (c and d) qPCR analysis of miR-1a, miR-133b, miR-206 (c), and their respective primary transcripts (d) in C2C12 cells transfected with siControl, siAkt1, or siAkt2 $(40 \mathrm{nM})$, and cultured either in GM or in DM for 2 days. The values shown are averages ( \pm S.E.M.) of three independent experiments performed in triplicate. (e) Northern blot analysis of total RNA purified from $\mathrm{C} 2 \mathrm{C} 12$ myoblasts cultured either in GM or DM (1 day) and then infected with either a control empty adenoviral vector (1d DM) or an adenoviral vector expressing constitutively active myristoylated AKT2 (1d DM + myr-AKT2) for further 2 days. Representative blots sequentially hybridized with miR-133, miR-1a, and U6 probes (left panels) or with miR-206, let-7a, and U6 probes (right panels) are displayed. (f) qPCR analysis of pri-miR-1a-2, pri-miR-133b, and pri-miR-206 in the RNA samples from C2C12 cells treated as in panel e. Statistical significance: ${ }^{* * \star} P<0.001$ (Student's $t$-test)

We transiently downregulated KSRP in myr-AKT2/GM C2C12 myoblasts (Supplementary Figure S3a). KSRP knockdown impaired the myr-AKT2-dependent induction of differentiation markers (Supplementary Figure $\mathrm{S} 3 \mathrm{~b}$ ) and the maturation of myogenic miRNAs (Figure 3a). We obtained similar results analyzing $\mathrm{C} 2 \mathrm{C} 12$ transfectants in which the expression of KSRP was stably downregulated by shRNA (Supplementary Figure S3c).

Using a primary miRNA in vitro processing assay ${ }^{27}$ we showed that extracts from both myr-AKT2/GM- and
DM-cultured $\mathrm{C} 2 \mathrm{C} 12$ myoblasts were able to process pri-miR-206 into pre-miR-206 in vitro, whereas extracts from C2C12 myoblasts cultured in GM were not (left panels in Supplementary Figures S3d and e). In order to investigate the possibility that phosphorylation by AKT directly affects the ability of KSRP to process pri-myomiRs to pre-myomiRs in vitro, either unphosphorylated or AKT2-phosphorylated, recombinant $\mathrm{KSRP}^{30}$ was added to extracts from $\mathrm{C} 2 \mathrm{C} 12$ myoblasts cultured in GM. Unphosphorylated KSRP was unable to promote pri-miR-206 and pri-miR-1a-2 processing 


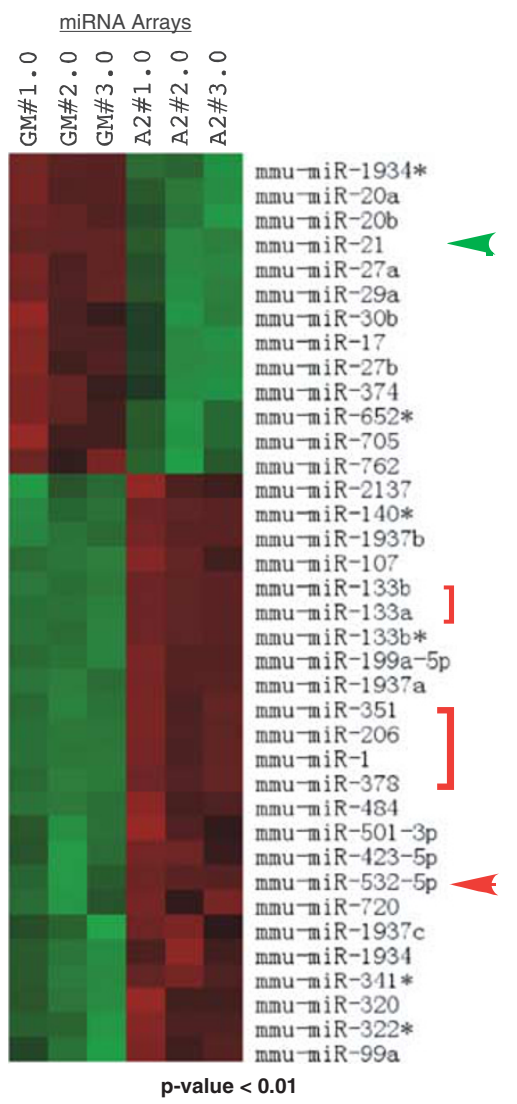

b gPCR
mature miRNAs Control $\square \quad$ primary miRNAs Control $\square$
mature miRNAs myr-AKT2 $\square$ primary miRNAs myr-AKT2
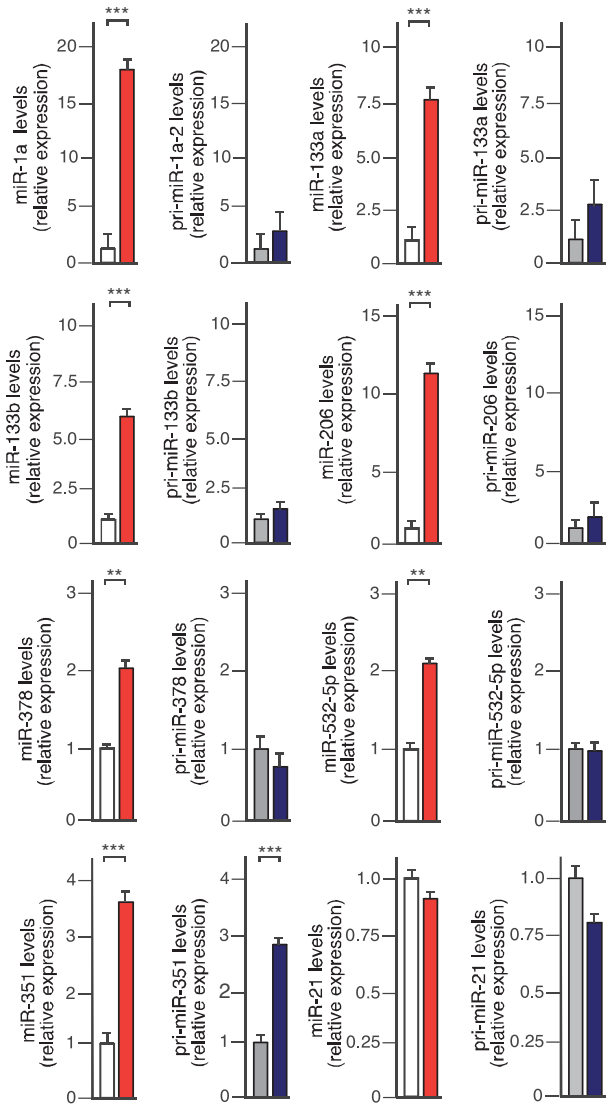

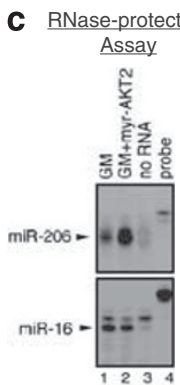

d

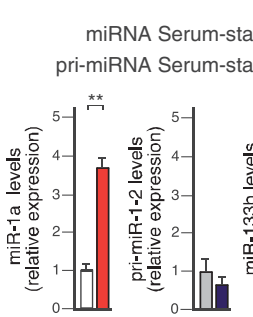

qPCR

e

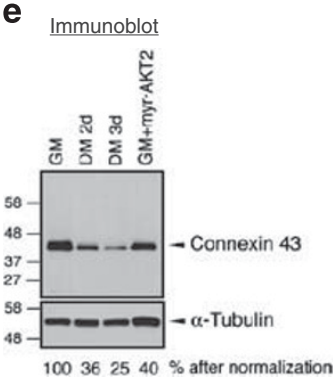

Figure 2 Identification and validation of miRNAs whose expression is induced by PI3K/AKT signaling in C2C12 myoblasts cultured in GM. (a) C2C12 myoblasts cultured in GM were infected with either a control empty adenoviral vector (GM no.;1.0, 2.0, and 3.0 represent three distinct experiments) or an adenoviral vector expressing myr-AKT2 (A2no:;1.0, 2.0, and 3.0 represent three distinct experiments) and harvested after 2 days. Total RNA was extracted and analyzed by miRNA microarrays. Green or red color on the heat map indicates a decrease or increase of miRNA levels, respectively, and color intensities correspond to relative signal levels on a logarithmic scale. The green arrow points to miR-21, whose expression was significantly reduced also by a 2-day treatment of $\mathrm{C} 2 \mathrm{C} 12$ myoblasts with DM. The red arrow and the red brackets indicate miRNAs whose expression was significantly enhanced also by a 2-day treatment of $\mathrm{C} 2 \mathrm{C} 12$ myoblasts with DM. Statistical analysis was performed using Student's t-test. (b) qPCR analysis of miRNAs and their respective primary transcripts (as indicated) in C2C12 myoblasts cultured in GM and infected with either a control or a myr-AKT2-expressing adenoviral vector. The values shown are averages ( \pm S.E.M.) of three independent experiments performed in triplicate. (c) RNase protection assay performed on the same RNA samples displayed in panel b. Samples were analyzed for the expression of either miR-206 or miR-16 (as control). Lanes corresponding to assays performed in the absence of RNA (no RNA) or in the presence of only RNA probes without digestion (probe) are also displayed. (d) qPCR analysis of miRNAs and their respective primary transcripts (as indicated) in $\mathrm{C} 2 \mathrm{C} 12$ myoblasts serum-starved for $16 \mathrm{~h}$ and cultured for two additional hours in the same medium in the absence or in the presence of $10^{-6} \mathrm{M}$ insulin. The values shown are averages ( \pm S.E.M.) of three independent experiments performed in triplicate. (e) Immunoblot analysis of total cell extracts from C2C12 cells cultured either in GM (and infected with control or myr-AKT2 vectors) or in DM (for 2 or 3 days). Blots were sequentially hybridized with anti-connexin 43 and anti-alpha-tubulin antibodies and films subjected to densitometric scanning. Densitometric values (\%) of connexin 43 expression after normalization for alpha-tubulin expression are presented. Representative blots are displayed. Statistical significance: ${ }^{* \star} P<0.01$ and ${ }^{\star \star \star} P<0.001$ (Student's $t$-test) 

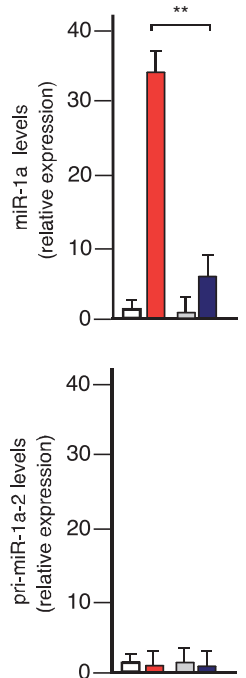

qPCR

siControl Ad-Control $\square$ sikSRP Ad-Control $\square$

siControl myr-AKT2 $\square$ siKSRP myr-AKT2 b
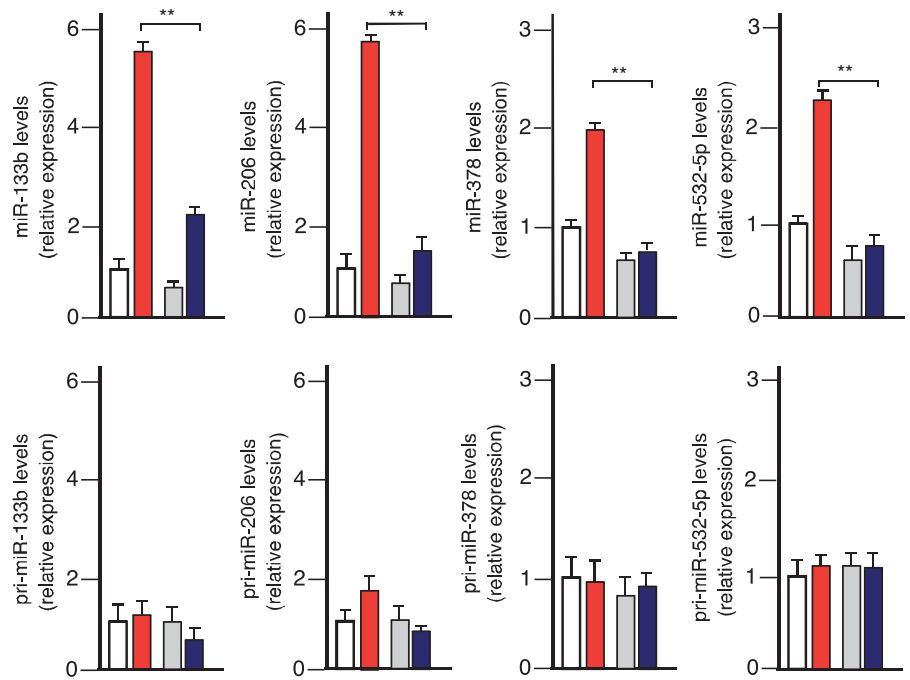

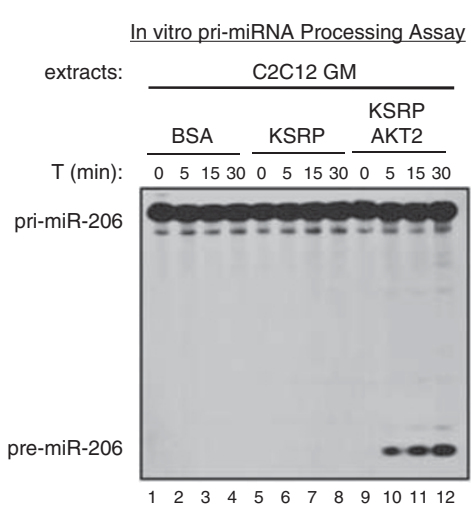

Figure 3 KSRP is required for AKT-induced myomiR maturation and phosphorylation by AKT activated KSRP ability to process pri-miR-206. (a) qPCR analysis of miRNAs (upper) and their respective primary transcripts (lower) in $\mathrm{C} 2 \mathrm{C} 12$ myoblasts cultured in GM, transfected with either siControl or siKsrp, and infected with either a control (Ad-Control) or a myr-AKT2-expressing adenoviral vector. The values shown are averages ( \pm S.E.M.) of three independent experiments performed in triplicate. (b) In vitro primiR-206 processing assays performed using total extracts from either C2C12 myoblasts cultured in GM pre-incubated with BSA (lanes 1-4), KSRP (30 nM, lanes 5-8), AKT2phosphorylated KSRP $\left(30 \mathrm{nM}\right.$, lanes 9-12), respectively. Internally ${ }^{32} \mathrm{P}$-labeled, pri-miR-206 RNA substrate was added and its processing monitored as described by Briata et al. ${ }^{8}$ Statistical significance: ${ }^{* \star} P<0.01$ (Student's $t$-test)

while in vitro phosphorylation by AKT2 activated the processing function of KSRP (Figure $3 \mathrm{~b}$ and data not shown). S193A mutation, which abrogates AKT phosphorylation, impairs the ability of AKT2 to activate the pri-miR-206 processing activity of GST-KH1-4 (a protein that includes the four $\mathrm{KH}$ domains of KSRP, comprises the AKT phosphorylation site ${ }^{30}$ and recapitulates KSRP activity to favor pri-miRNA processing) (Supplementary Figure S4).

These results suggest the existence of a PI3K/AKT > KSRP pathway that activates maturation of myomiRs in $\mathrm{C} 2 \mathrm{C} 12$ myoblasts.

KSRP knockout impairs myomiR maturation in the course of muscle regeneration. We have recently generated Ksrp knock-out (Ksrp ${ }^{-1-}$, Supplementary Figure S5a and b) mice that have been described elsewhere. ${ }^{36}$ Adult skeletal muscle regenerates in response to injuries with a process in which PI3K/AKT signaling activation has an essential role (reviewed in Schiaffino and Mammucari ${ }^{37}$ and Supplementary Figure S1a). As the regenerative process has been recently linked to induction of myomiR expression $^{38}$ we asked whether Ksrp knockout affects miomyR processing in injury-dependent muscle regeneration.

In order to evaluate the expression of myomiRs during muscle regeneration, both $\mathrm{Ksrp}^{-1-}$ and their wild-type (WT) littermates were injected with cardiotoxin in the tibialis anterioris muscle and sacrificed at different times (Supplementary Figures S5c). The analysis of proliferation/differentiation markers in regenerating muscles revealed a stronger and more sustained induction of Ccna2 (also known as
Cyclin A2) in $\mathrm{Ksrp}^{-1-}$ in comparison with WT mice (Supplementary Figures S5d). Conversely, myogenin induction at day 4 after injury was significantly impaired in $\mathrm{Ksrp}^{-1-}$ in comparison with WT mice (Supplementary Figure S5e). Hematoxylin-eosin staining of cross-sections of non-injured (day 0 ) and injured muscles showed dishomogeneous and hypernucleated regenerating myofibers at day 7 after cardiotoxin injection in $\mathrm{Ksrp}^{-1-}$ mice (Supplementary Figure S5f).

As shown in Supplementary Figure S5g and in the left panel of Figure 4a, miR-206 expression transiently declined at day 2 post-injection in both WT and $\mathrm{Ksrp}^{-1-}$ mice and strongly increased starting from day 4 to day 14 in WT animals. miR206 increase was significantly reduced in $\mathrm{Ksrp}^{-1-}$ mice when compared with WT animals (Figure 4a, left panel). miR-133b and miR-1a expression levels sharply decreased between days 2 and 7 while almost reached pre-injection levels at day 14 (Figure 4a, middle and right panels). A significant reduction of the expression of miR-133b and miR-1a was detectable at day 14 in $\mathrm{Ksrp}^{-1-}$ mice when compared with WT (Figure 4a, middle and right panels). Importantly, pri-miR-206 and pri-miR-133b induction, occurring at day 4 post-injury, was significantly higher in $\mathrm{Ksrp}^{-1-}$ than in WT mice suggesting an accumulation of unprocessed primary myomiRs (Figure $4 \mathrm{~b}$, left and middle panels). Also pri-miR-1a-2 levels, although with a different kinetic compared with pri-miR-206 and pri-miR-133b, were significantly higher at day 4 in $\mathrm{Ksrp}^{-/-}$ mice when compared with WT animals (Figure 4b, right panel).

Altogether, these data indicate that the induction of myomiRs occurring during muscle regeneration is impaired in $\mathrm{Ksrp}^{-1-}$ mice due to maturation blockade. 
a

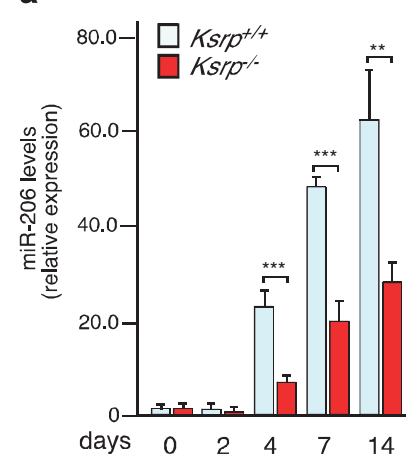

b




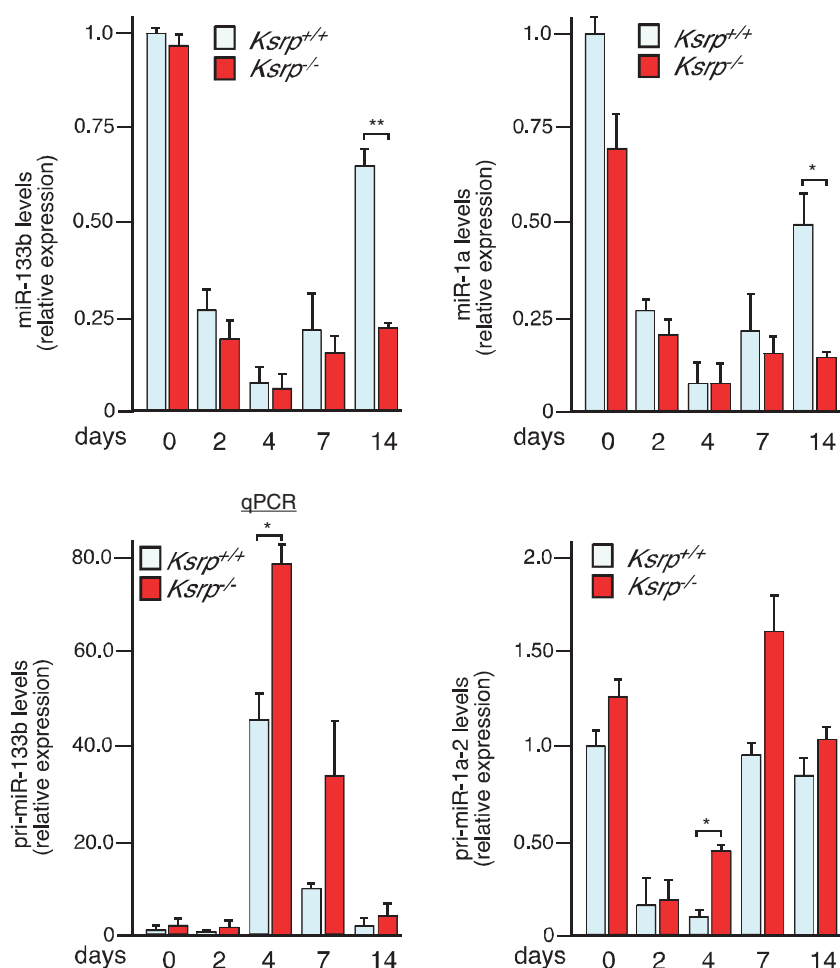



Figure 4 Impaired maturation of myomiRs during muscle regeneration in Ksrp knock-out mice. (a and $\mathbf{b})$ qPCR analysis of miRNAs (a) and their respective primary transcripts (b) in total RNA samples extracted from tibialis anterioris muscles of either wild-type (Ksrp ${ }^{+1+}$ ) or $\mathrm{Ksrp}^{-1-}$ mice before and at different intervals of time after injection of cardiotoxin as indicated. The values shown are averages ( \pm S.D.) of five independent animals for each experimental group. Assays were performed in triplicate. Statistical significance: ${ }^{*} P<0.05$, ${ }^{\star \star} P<0.01$, and ${ }^{\star \star \star} P<0.001$ (Student's $t$-test)

PI3K/AKT inhibits KSRP ability to promote decay of myogenin mRNA while promotes its recruitment to myogenic miRNA processing complexes. We and others have shown that stabilization of inherently labile muscle specific transcripts takes place during $\mathrm{C} 2 \mathrm{C} 12$ cell differentiation. ${ }^{6-8}$ Myogenin and p21 mRNAs are unstable in C2C12 myoblasts cultured in GM and KSRP is required for their decay while MAPK p38-induced inactivation of KSRP (which occurs in differentiating myoblasts) contributes to myogenin and p21 induction. ${ }^{8}$ Figure $5 a$ and Supplementary Figure S6a show that AKT increased the half life of myogenin and p21 mRNAs when activated in C2C12 myoblasts cultured in GM. On the basis of this observation and the above data, we hypothesized that PI3K/AKT signaling activation reduces KSRP ability to promote mRNA decay while induces its ability to promote miRNA precursor maturation in the course of $\mathrm{C} 2 \mathrm{C} 12$ myoblasts differentiation.

To address this hypothesis, we first performed in vitro mRNA degradation assays supplementing extracts from C2C12 myoblasts cultured in DM with purified recombinant KSRP. ${ }^{8}$ In vitro phosphorylation by AKT2 impairs the decaypromoting activity of KSRP on a myogenin mRNA substrate (Figure 5b), thus indicating that, besides inducing a subcellular re-distribution of KSRP (Supplementary Figure S6b, and Briata et al. ${ }^{26}$ ), phosphorylation by AKT2 directly affects KSRP function on myogenin mRNA decay.
This finding, together with the observation that phosphorylation by AKT2 enhances the ability of KSRP to promote pri-miR-206 processing (Figure $3 b$ ) prompted us to address the possibility that PI3K/AKT activation in $\mathrm{C} 2 \mathrm{C} 12$ myoblasts displaces KSRP from myogenic mRNA destabilizing complexes and relocates it to primary myomiR processing complexes. Indeed, ribonucleoprotein immunoprecipitation (RIP) assays show that PI3K/AKT activation in C2C12 cells, as well as culture in DM, caused dissociation of KSRP from myogenin mRNA while it increased KSRP interaction with primiR-133b, pri-miR-206, and pri-miR-532-5p (Figures 5c and d and data not shown). Finally, we investigated whether PI3K/ AKT activation affects KSRP ability to interact with the enzymatic complexes responsible for either labile mRNA decay or pri-miRNA processing. Indeed, once phosphorylated by AKT2, KSRP was unable to interact with the exosome complex (represented by EXOSC10 component, Figure 5e) while became capable to interact with the Drosha complex (Figure 5f). As a control for AKT2-dependent inhibition of KSRP interaction with the Exosome, we showed that the exosome-binding ability of a recombinant KSRP mutated in the critical Ser $193^{30}$ was unaffected by AKT2 phosphorylation (Figure 5e).

Altogether these data suggest that phosphorylation by AKT displaces KSRP from myogenic mRNA-destabilizing complexes, relocating it to primary myomiR-processing complexes. 
a

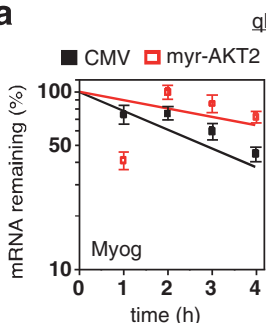

gPCR

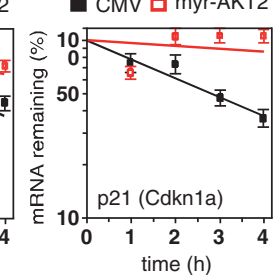

C

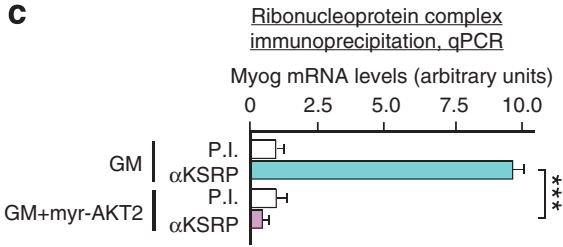

d Ribonucleoprotein complex immunoprecipitation, QPCR pri-miR-133b levels (arbitrary units)

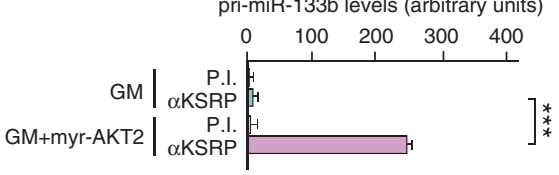

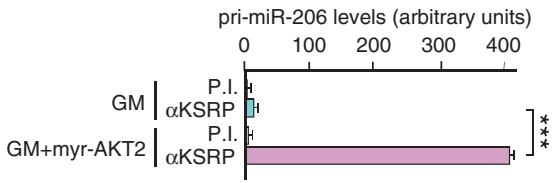

pri-miR-532-5p levels (arbitrary units)

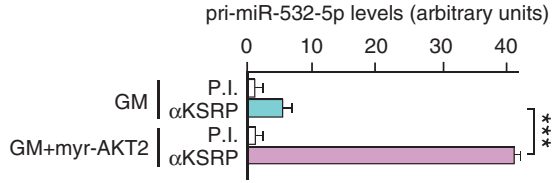

b

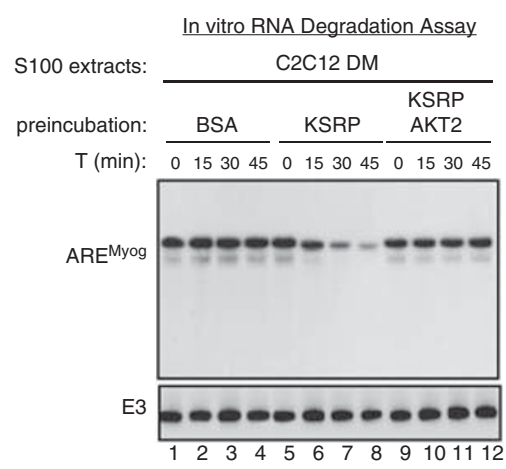

e GST Pulldown, Immunoblot

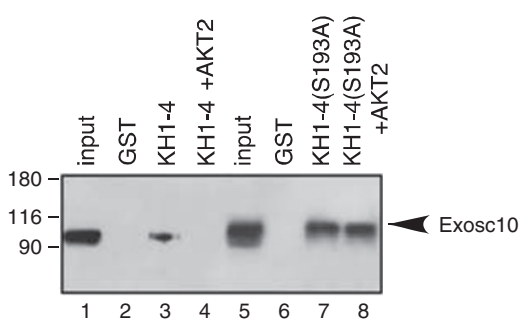

f

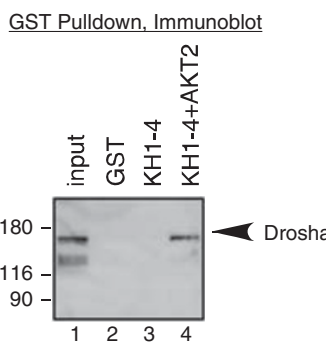

Figure 5 AKT signaling activation inhibits KSRP ability to participate in a Myogenin mRNA decay complex while favors its association with a primary myomiR processing complex. (a) qPCR analysis of myogenin and p21 (also known as Cdkn1a) expression in C2C12 myoblasts cultured in GM and infected with either a control (GM) or a myr-AKT2-expressing adenoviral vector. Total RNA was isolated at the indicated times after the addition of DRB. The values shown are averages ( \pm S.E.M.) of three independent experiments performed in triplicate. (b) In vitro RNA degradation assays using S100 extracts from C2C12 myoblasts cultured in DM pre-incubated with BSA (lanes 1-4), KSRP (30 nM, lanes 5-8), AKT2-phosphorylated KSRP (30 nM, lanes 9-12), respectively. Internally ${ }^{32}$ P-labeled and capped RNA substrates were added and their decay monitored as described under Material and Methods. E3 is a stable unrelated transcript. ${ }^{9}$ Representative gels are displayed. (c) C2C12 myoblasts were cultured in GM and infected with either control (GM) or myr-AKT2-expressing adenoviral vector. After 2 days cells were lysed, and total cell extracts were immunoprecipitated as indicated. RNA was purified from immunocomplexes and analyzed by qPCR to detect Myogenin mRNA. The values shown are averages ( \pm S.E.M.) of three independent experiments performed in triplicate. (d) $\mathrm{C} 2 \mathrm{C} 12$ myoblasts were cultured in $\mathrm{GM}$ and infected with either control (GM) or myr-AKT2-expressing adenoviral vector. After 2 days cells were lysed, and total cell extracts were immunoprecipitated as indicated. RNA was purified from immunocomplexes and analyzed by qPCR to detect primary myomiRs as indicated. The values shown are averages ( \pm S.E.M.) of three independent experiments performed in triplicate. (e and f) GST pull-down of endogenous Exosc10 (e) and Drosha (f) present in total extracts from C2C12 cells cultured in GM using the following proteins: GST, GST-KH1-4, GST-KH1-4 in vitro phosphorylated by AKT2, GST-KH14(S193A), or GST-KH1-4(S193A) in vitro phosphorylated by AKT2 as indicated. GST-KH1-4(S193A) is mutated in Ser 193 in order to abolish the AKT phosphorylation site as described by Gherzi et al. ${ }^{30}$ Proteins were analyzed by immunoblotting using the indicated antibodies. Representative blots are displayed. Statistical significance: ${ }^{* * \star} P<0.001$ (Student's $t$-test)

\section{Discussion}

In this manuscript we report that PI3K/AKT signaling activation occurring at early phases of C2C12 myoblast differentiation induces the expression of myomiRs activating their processing from primary transcripts with KSRP having a required role in this pathway. AKT regulates in opposite ways two distinct KSRP functions inhibiting its ability to promote decay of myogenin mRNA and activating its ability to favor maturation of myomiRs from precursors. This dynamic regulatory switch eventually contributes to the activation of the myogenic program.

PI3K/AKT signaling has been reported to regulate the expression of some miRNAs in fibroblast and epithelial cells ${ }^{39}$ while a role for the pathway in controlling miRNA maturation has been only postulated. ${ }^{40}$ Our data indicate that either physiological or forced activation of PI3K/AKT favors the processing of primary myomiRs leading to accumulation of their mature forms. We and Others have shown that KSRP promotes processing of a select group of miRNAs, including 
the let-7 family, in HeLa and other cell lines. ${ }^{27,29,41}$ KSRP silencing in $\mathrm{C} 2 \mathrm{C} 12$ myoblasts downregulates the expression of the above miRNAs (Supplementary Figure S6c), thus indicating that an overlap between miRNAs, whose expression is affected by KSRP knockdown in HeLa and C2C12 cells, does exist. However, the overlap is partial as some KSRP-regulated miRNAs expressed in $\mathrm{C} 2 \mathrm{C} 12$ myoblasts are not expressed in HeLa cells (miR-1a, miR-133a, miR-133b, miR-206, and miR-378) and, conversely, other KSRPregulated miRNAs are expressed in HeLa cells but not in C2C12 myoblasts (miR-335 and miR-595) (according to miRNA microarray analyses performed in this study and in Trabucchi et al. ${ }^{27}$ ). KSRP interacts in $\mathrm{C} 2 \mathrm{C} 12$ myoblasts with myogenic (Figure $5 \mathrm{~d}$ ), as well as with non-myogenic primiRNAs (Supplementary Figure S6d). However, PI3K/AKT activation in $\mathrm{C} 2 \mathrm{C} 12$ cells strongly enhances KSRP interaction with myogenic pri-miRNAs (Figure 5d), as well as their processing into miRNAs (Figure $2 b$ ) while it does not increase either KSRP interaction with non-myogenic pri-miRNA or the expression of the corresponding miRNAs (Supplementary Figure S6d, e, and Figure 2b). It is also noteworthy that maturation of primary let-7a-1 is constitutive in $\mathrm{C} 2 \mathrm{C} 12$ myoblasts and is not affected by either DM or myr-AKT2/ GM (right panels in Supplementary Figures S3d and e). On the basis of these data, we are tempted to hypothesize that phosphorylation by AKT might increase the ability of KSRP to interact with ribonucleoprotein complexes specifically comprising myomiR precursors. This could be achieved through the interaction of AKT-phosphorylated KSRP with a specific co-activator of myogenic pri-miRNA processing.

Studies performed in cultured myoblasts and in animals have demonstrated that myomiRs regulate fundamental aspects of myoblast biology such as differentiation and proliferation. ${ }^{10,11}$ However, the molecular mechanisms by which the expression of myomiRs is dynamically regulated by activation of distinct signaling pathways during skeletal myogenesis have not been elucidated. Our data highlight the relevance of PI3K/AKT signaling as an exquisite regulator of myomiR maturation. Noticeably, culture in DM, differently from activation of AKT signaling in GM, strongly induces primiR-133b and pri-miR-206 expression and moderately enhances pri-miR1a-2 levels (Figure 1b). PI3K/AKT pathway inhibition in $\mathrm{C} 2 \mathrm{C} 12$ myoblasts cultured in DM does not reduce the expression of primary myomiRs (Figures $1 b$ and $d$ ) while strongly impairs their maturation (Figures 1a and c). This is in agreement with previous data from different laboratories demonstrating the transcriptional regulation of primary myomiR levels upon myoblast exposure to DM (reviewed in Williams et $a .^{10}$ ). Altogether, these observations and our findings suggest that a stringent control of myomiR expression occurs during myogenesis at different regulatory levels, with PI3K/AKT signaling being central in the control of maturation.

As other single-strand RNA binding proteins, KSRP proved to be involved at different levels in RNA metabolism with its ability to promote labile mRNA decay, as well as miRNA precursor maturation as the most extensively studied functions. ${ }^{26}$ However, despite the evidence that KSRP is targeted by distinct signaling pathways, ${ }^{8,29,30}$ the possibility that a specific signal induces a biological effect by regulating both
KSRP main functions in an integrated and dynamic way has never been addressed. C2C12 myoblasts, an established culture model of muscle satellite cells that we have previously utilized to demonstrate KSRP function on labile myogenic mRNA decay, ${ }^{8}$ were ideal to investigate this possibility. Indeed, phosphorylation by AKT inhibits KSRP ability to interact with the Exosome complex and to promote decay of Myogenin mRNA while activates KSRP ability to interact with Drosha complex and to favor the processing of myogenic miRNAs.

Although studies on cultured muscle cells allowed us to identify the $\mathrm{PI} 3 \mathrm{~K} / \mathrm{AKT}>\mathrm{KSRP}>$ myomiR processing pathway, we obtained definitive evidence of the physiological relevance of KSRP in primary myomiR processing using the Ksrp knock-out mice that display an impaired myomiR maturation during injury-induced muscle regeneration. However, the effect of Ksrp knockout on myoblast proliferation and differentiation markers, as well as on myofibers morphology is transient. One possible explanation is that besides PI3K/AKT signaling, other pathways are activated during the course of muscle regeneration. ${ }^{5}$ These pathways, targeting effector proteins different from KSRP, can modulate the molecular events implicated in muscle regeneration in a KSRPindependent way and, in principle, compensate for the molecular defects due to KSRP ablation. Another possibility is that Ksrp gene deletion leads to the overexpression of RNA binding proteins (some have been previously described as post-transcriptional targets of KSRP (reviewed in Briata et al. $\left.^{26}\right)$ ) that could be able to compensate for some of the KSRP functions in mice lacking Ksrp. Further studies will be required to investigate the consequences of KSRP deletion and the reduced myomiR expression on the function of regenerated muscles.

$\mathrm{PI}$ KK/AKT signaling affects myogenesis at different levels, regulating chromatin remodeling at myogenic loci and controlling Cyclin D1 expression at post-transcriptional level. ${ }^{5,9}$ Our present data reveal an unanticipated additional effect elicited by PI3K/AKT signaling, which dynamically integrates two KSRP functions contributing to the post-transcriptional control of skeletal myogenesis.

\section{Materials and Methods}

Cell cultures, transfections, plasmids and adenoviral infections. Murine C2C12 myoblasts (obtained from ATCC, no. CRL-1772, Lot no. 58236521) were cultured in DMEM plus 20\% FBS (Growth Medium, GM). Myogenic differentiation was induced by incubation in DMEM plus $2 \%$ horse serum (Differentiation Medium, DM). For transient transfections, $\mathrm{C} 2 \mathrm{C} 12$ cells were electroporated using the Nucleofector II, according to manufacturer's instructions (Amaxa, Walkersville, MD, USA). C2C12 cell transfections aimed to obtain stable shKSRP transfectants were performed using Lipofectamine Plus (Invitrogen, Carlsbad, CA, USA) and Puromycin (Invivogen, San Diego, CA, USA) was used at $2.5 \mu \mathrm{g} / \mathrm{ml}$ for selection. Pools of transfected cells were used for experiments. The adenoviral vectors pAdCMVnull (Ad-Control) and pAdmyrAKT2 (myr-AKT2) were purchased from Vector Biolabs (Eagleville, PA, USA) and used as previously described. $^{8}$

Recombinant proteins and antibodies. Production and purification of recombinant GST-KH1-4 (comprising the four KH domain of human KSRP) were performed as previously described. ${ }^{8}$ Baculovirus-based production and purification of His-tagged human KSRP were previously described ${ }^{8}$ Affinity-purified rabbit polyclonal anti-KSRP antibody was previously described. ${ }^{8}$ Affinity-purified rabbit polyclonal anti-Drosha was from Abcam (Cambridge, UK). Affinity-purified 
rabbit polyclonal anti-AKT and anti-Phospho-AKT (Ser 473) were from Cell Signaling (Danvers, MA, USA). Affinity-purified rabbit polyclonal anti-EXOSC10, mouse monoclonal antibody anti-alpha-Tubulin, and rabbit polyclonal antibodies anti-connexin 43 were from Sigma (St. Louis, MO, USA).

SiRNA and shRNA-mediated knockdown. siRNA utilized to knockdown Ksrp expression were described previously. ${ }^{8}$ In order to knockdown the expression of either Akt1 or Akt2, we utilized siRNAs purchased from Santa Cruz (Santa Cruz, CA, USA).

In vitro kinase assays. AKT2 kinase assays were performed using the preactivated enzyme purchased from Millipore (Billerica, MA, USA) as recommended by manufacturer. We have previously demonstrated that both AKT1 and AKT2 phosphorylate to a similar extent recombinant KSRP. ${ }^{30}$

miRNA profiling. MicroRNA microarray assays were provided by LC Sciences (Houston, TX, USA). Total RNA was prepared from either control-infected (cultured in GM or in DM) or myr-AKT2-infected (cultured in GM) C2C12 myoblasts using Trizol (Invitrogen), enriched with RNA smaller than 40 nt using PureLink miRNA Isolation Kit (Invitrogen) and used to interrogate a HumanMouseRat miRNA Array (LC Sciences) comprising a total of 17341 mature miRNA sequences, based on Sanger miRBase release 16.0. Data were analyzed by first subtracting the background and then normalizing the signals using a LOWESS filter (locally-weighted regression performed at LC Sciences). The ratio of the two sets of detected signals (log2 transformed and balanced) and $P$-values of the $t$-test were calculated; differentially detected signals were those with $<0.01 P$-values.

Quantitative RT-PCR. Total RNA was isolated using the miRNeasy mini kit (Qiagen, Milano, Italy), treated with DNAsel (Promega, Madison, WI, USA), and retro-transcribed using miScript reverse transcription kit (Qiagen) according to manufacturers' instructions. For quantitative RT-PCR (qPCR), $100 \mathrm{ng}$ of DNAse I-treated total RNA were retro-transcribed and PCR reactions were performed using the miScript SYBR green PCR kit (Qiagen) and the Realplex II Mastercycler (Eppendorf, Milano, Italy). The sequence-specific primers used for PCR reactions are listed in Supplementary Table S1.

Northern Blot and RNase-protection assays. Total RNA (10 $\mu \mathrm{g} / \mathrm{lane})$ was resolved on $15 \%$ polyacrylamide-urea gels, and electroblotted onto HyBond $\mathrm{N}+$ membranes (GE Healthcare, Buckinghamshire, England). Membranes were hybridized overnight with radiolabeled antisense miRNAs in ExpressHyb solution (Clontech, Mountain View, CA, USA). After hybridization, membranes were washed three times with $2 \times$ SSC and $0.05 \%$ SDS, twice with $0.1 \times$ SSC and $0.1 \%$ SDS, exposed overnight to imaging screens, and analyzed using a Storm 860 Phosphorlmager (GE Healthcare). The same blot was hybridized (upon stripping in boiling $0.1 \%$ SDS) with three distinct probes, including control U6 RNA.

RNase protection assays were performed using the mirVana miRNA Probe Contruction and mirVana miRNA Detection kits from Ambion (Austin, TX, USA) according to manufacturers' instructions.

Pri-miRNA in vitro processing assays. For pri-miRNA processing assays, total cell extracts from $\mathrm{C} 2 \mathrm{C} 12$ myoblasts were prepared in $50 \mathrm{mM}$ Tris- $\mathrm{HCl}(\mathrm{pH} 8.0)$, $150 \mathrm{mM} \mathrm{NaCl}, 0.5 \%$ Triton X-100, $1 \times$ Complete (Roche, Mannheim, Germany), and $10 \%$ glycerol) and incubated (typically $40 \mu \mathrm{g}$ per $25 \mu$ l reaction at $37^{\circ} \mathrm{C}$ for the indicated times) with in-vitro synthesized and uniformly labeled pri-miRNA ( $5 \mathrm{fmol})$ in processing buffer containing $100 \mathrm{mM} \mathrm{KCH}{ }_{3} \mathrm{COOH}, 2 \mathrm{mM} \mathrm{Mg}\left(\mathrm{CH}_{3} \mathrm{COOH}\right)_{2}, 10 \mathrm{mM}$ Tris-Cl (pH 7.6), $2 \mathrm{mM}$ DTT, $10 \mathrm{mM}$ creatine phosphate, $1 \mu \mathrm{g}$ of creatine phosphokinase, $1 \mathrm{mM}$ ATP, $0.4 \mathrm{mM}$ GTP, $0.1 \mathrm{mM}$ spermine, and 2 units of Stop RNase Inhibitor (5 PRIME, Hamburg, Germany).

RNA in vitro degradation. ${ }^{32} \mathrm{P}$-labeled RNAs were synthesized and used as substrates for in vitro degradation assays as reported..$^{8}$

RNA in vitro degradation assay and measure of mRNA half-life in intact cells. ${ }^{32}$ P-labeled. RNAs were synthesized and used as substrates for in vitro degradation assays as reported. ${ }^{8}$ ARE ${ }^{\text {Myog }}$ and the E3 sequence from Pitx2 3'UTR were described previously. ${ }^{8,9}$

To measure the mRNA half-life, intact $\mathrm{C} 2 \mathrm{C} 12$ myoblasts were treated with $100 \mu \mathrm{M}$ DRB (5,6-dichloro-1- $\beta$-D-ribofuranosylbenzimidazole, 5,6-dichlorobenzimidazole riboside), harvested at the indicated times, and total RNA was isolated and analyzed as above.

Ribonucleoprotein complexes immunoprecipitation (RIP) assays. RIP assays were performed as previously described in Trabucchi. ${ }^{27}$ Briefly, cell lysates were immunoprecipitated with protein A-coupled antibodies at $4{ }^{\circ} \mathrm{C}$ overnight. Pellets were sequentially washed with the following buffers: Buffer I (0.1\% SDS, $1 \%$ Triton X-100, $2 \mathrm{mM}$ EDTA, $20 \mathrm{mM}$ Tris- $\mathrm{HCl}$, pH 8.1, and $150 \mathrm{mM}$ $\mathrm{NaCl})$; Buffer II (0.1\% SDS, 1\% Triton X-100, 2 mM EDTA, 20 mM Tris-HCl, pH 8.1, and $500 \mathrm{mM} \mathrm{NaCl})$; and Buffer III (0.25 M LiCl, $1 \% \mathrm{NP}-40,1 \%$ deoxycholate, $1 \mathrm{mM}$ EDTA, $10 \mathrm{mM}$ Tris- $\mathrm{HCl}$, and $\mathrm{pH}$ 8.1). Total RNA was prepared from immunocomplexes using Trizol (Invitrogen) retro-transcribed and amplified by qPCR as described above. The primer sequences are detailed in Supplementary Table S1.

Nuclear and S100 extracts preparation. Nuclear and S100 extracts were prepared as previously described. ${ }^{8}$

GST pull-down assays. Escherichia coli, transformed with pGEX-based recombinant plasmids (GE Healthcare) were grown until the $A_{600}$ reached 0.6, induced with $1 \mathrm{mM}$ of isopropyl- $\beta$-D-thiogalactoside (IPTG) for $3 \mathrm{~h}$, harvested, washed with PBS, and lysed by sonication. After centrifugation $(14000 \times g$ for $15 \mathrm{~min}$ at $4^{\circ} \mathrm{C}$ ) the supernatants were incubated with glutathione-sepharose beads (GE Healthcare) for $1 \mathrm{~h}$ at $4{ }^{\circ} \mathrm{C}$ under rotation. Beads were washed four times with pull-down buffer (PDB, $150 \mathrm{mM} \mathrm{NaCl}, 20 \mathrm{mM}$ Tris-HCl pH 8.0, $0.2 \%$ Nonidet P-40, 10\% glycerol, and a mixture of protein inhibitors (Complete, Roche), saturated with bovine serum albumin, washed again with PDB, and incubated with $300 \mu \mathrm{g}$ of cell extracts $\left(2 \mathrm{~h}, 4^{\circ} \mathrm{C}\right.$, and rotating). Beads were collected by centrifugation $(30 \mathrm{~s}, 5000 \times \mathrm{g})$ and washed four times with PDB. Bound proteins were eluted in SDS sample buffer, resolved by SDS-PAGE, and analyzed by Immunoblot. Plasmids GST-KH1-4 and GST-KH1-4(S193) were described previously. ${ }^{30}$

Generation of Ksrp-null mice. Generation of Ksrp-null mice $\left(\mathrm{Ksrp}^{-1-}\right)$ is described elsewhere. ${ }^{36}$ Briefly, a hygromycin cassette flanked by loxP sites was designed to replace exon 1 to exon 13 of Ksrp gene and electroporated into V6.5 mouse embryonic stem (ES) cells. The cells were subsequently selected by hygromycin $(250 \mu \mathrm{g} / \mathrm{ml})$ and ganciclovir $(2 \mu \mathrm{M})$. ES colonies resistant to hygromycin and ganciclovir were genotyped by Southern blot analysis, and the Ksrp-targeted ES clones, after Cre recombinase-mediated removal of the hygromycin cassette, were injected into $\mathrm{C} 56 \mathrm{BL} / 6$ blastocysts to generate chimera mice. Male chimeras were further backcrossed with $\mathrm{C} 56 \mathrm{BL} / 6$ females for eight generations and were then used for the experiments. All animal studies were conducted in accordance with guidelines for animal use and care established by the University of Alabama at Birmingham Animal Resource Program and the Institutional Animal Care and Use Committee.

Injury-induced muscle regeneration. Animals were anesthetized by inhalation of Isofluorane and the site of injection was shaved and sterilized according to the animal care requirements. $25 \mu \mathrm{l}$ of $10 \mu \mathrm{M}$ Cardiotoxin (Latoxan, Valence, France) (in sterile PBS) was slowly injected into the tibialis anterioris muscle. Animals were euthanized at different time points after injection ( $0-14$ days) using $\mathrm{CO}_{2}$ asphyxiation, and the musculature of both lower limbs was removed and processed for Hematoxylin-eosin staining and RNA preparation.

\section{Conflict of Interest}

The authors declare no conflict of interest.

Acknowledgements. Part of the studies has been conducted in the laboratories and facilities of the Centro Biotecnologie Avanzate (CBA, Genova, Italy). This work has been partly supported by Associazione Italiana per la Ricerca sul Cancro (AIRC), Association for International Cancer research (AICR grant no. 10-0527) and Limonte 2 (Regione Liguria, RNA Technology) to RG; the work of MP is supported by AICR grant no. 10-0527. We are indebted with Drs. Imed E Gallouzi (McGill University) for providing us the cardiotoxin protocol and Zhenguo Wu (Hong Kong University of Science \& Technology) for useful discussions. 
1. Yun K, Wold B. Skeletal muscle determination and differentiation: story of a core regulatory network and its context. Curr Opin Cell Biol 1996; 8: 877-889.

2. Charge SB, Rudnicki MA. Cellular and molecular regulation of muscle regeneration. Physiol Rev 2004; 84: 209-238.

3. Tedesco FS, Dellavalle A, Diaz-Manera J, Messina G, Cossu G. Repairing skeletal muscle: regenerative potential of skeletal muscle stem cells. J Clin Invest 2010; 120: 11-19.

4. Tajbakhsh S. Skeletal muscle stem cells in developmental versus regenerative myogenesis. J Intern Med 2009; 266: 372-389.

5. Guasconi V, Puri PL. Chromatin: the interface between extrinsic cues and the epigenetic regulation of muscle regeneration. Trends Cell Biol 2009; 19: 286-294.

6. van der Giessen K, Di-Marco S, Clair E, Gallouzi IE. RNAi-mediated HuR depletion leads to the inhibition of muscle cell differentiation. J Biol Chem 2003; 278: 47119-47128.

7. Figueroa A, Cuadrado A, Fan J, Atasoy U, Muscat GE, Muñoz-Canoves $P$ et al. Role of HuR in skeletal myogenesis through coordinate regulation of muscle differentiation genes. Mol Cell Biol 2003; 23: 4991-5004.

8. Briata $\mathrm{P}$, Forcales SV, Ponassi M, Corte G, Chen CY, Karin M et al, p38-dependent phosphorylation of the mRNA decay-promoting factor KSRP controls the stability of select myogenic transcripts. Mol Cell 2005; 20: 891-903.

9. Gherzi R, Trabucchi M, Ponassi M, Gallouzi IE, Rosenfeld MG, Briata P. Akt2-mediated phosphorylation of Pitx2 controls Cond1 mRNA decay during muscle cell differentiation. Cell Death Differ 2010; 17: 975-983.

10. Williams AH, Liu N, van Rooij E, Olson EN. MicroRNA control of muscle development and disease. Curr Opin Cell Biol 2009; 21: 461-469.

11. Güller I, Russell AP. MicroRNAs in skeletal muscle: their role and regulation in development, disease and function. J Physiol 2010; 588: 4075-4087.

12. Chen JF, Mandel EM, Thomson JM, Wu Q, Callis TE, Hammond SM et al. The role of microRNA-1 and microRNA-133 in skeletal muscle proliferation and differentiation. Nat Genet 2006; 38: 228-233.

13. Kim HK, Lee YS, Sivaprasad U, Malhotra A, Dutta A. Muscle-specific microRNA miR-206 promotes muscle differentiation. J Cell Biol 2006; 174: 677-687.

14. Rao PK, Kumar RM, Farkhondeh M, Baskerville S, Lodish HF. Myogenic factors that regulate expression of muscle-specific microRNAs. Proc Natl Acad Sci USA 2006; 103 8721-8726

15. Taulli R, Bersani F, Foglizzo V, Linari A, Vigna E, Ladanyi $M$ et al. The muscle-specific microRNA miR-206 blocks human rhabdomyosarcoma growth in xenotransplanted mice by promoting myogenic differentiation. J Clin Invest 2009; 119: 2366-2378.

16. Elia L, Contu R, Quintavalle M, Varrone F, Chimenti C, Russo MA et al. Reciproca regulation of microRNA-1 and insulin-like growth factor-1 signal transduction cascade in cardiac and skeletal muscle in physiological and pathological conditions. Circulation 2009; 120: $2377-2385$.

17. Cacchiarelli D, Martone J, Girardi E, Cesana M, Incitti T, Morlando M et al. MicroRNAs involved in molecular circuitries relevant for the Duchenne muscular dystrophy pathogenesis are controlled by the dystrophin/nNOS pathway. Cell Metab 2010; 12 : 341-351.

18. Krol J, Loedige I, Filipowicz W. The widespread regulation of microRNA biogenesis function and decay. Nat Rev Genet 2010; 11: 597-610.

19. Fabian MR, Sonenberg N, Filipowicz W. Regulation of mRNA translation and stability by microRNAs. Annu Rev Biochem 2010; 79: 351-379.

20. Newman MA, Hammond SM. Emerging paradigms of regulated microRNA processing Genes Dev 2010; 24: 1086-1092.
21. Thomson JM, Newman M, Parker JS, Morin-Kensicki EM, Wright T, Hammond SM. Extensive post-transcriptional regulation of microRNAs and its implications for cancer. Genes Dev 2006; 20: 2202-2207.

22. Kumar MS, Lu J, Mercer KL, Golub TR, Jacks T. Impaired microRNA processing enhances cellular transformation and tumorigenesis. Nat Genet 2007; 39: 673-677.

23. O'Rourke JR, Georges SA, Seay HR, Tapscott SJ, McManus MT, Goldhamer DJ et al. Essential role for Dicer during skeletal muscle development. Dev Biol 2007; 311: 359-368.

24. Paroo Z, Ye X, Chen S, Liu Q. Phosphorylation of the human microRNA-generating complex mediates MAPK/Erk signaling. Cell 2009; 139: 112-122.

25. Trabucchi M, Briata P, Filipowicz W, Rosenfeld MG, Ramos A, Gherzi R. How to control miRNA maturation? RNA Biol 2009; 6: 536-540.

26. Briata $\mathrm{P}$, Chen $\mathrm{CY}$, Giovarelli M, Pasero M, Trabucchi M, Ramos A et al. KSRP, many functions for a single protein. Front Biosci 2011; 16: 1787-1796.

27. Trabucchi M, Briata P, Garcia-Mayoral M, Haase AD, Filipowicz W, Ramos A et al. The RNA-binding protein KSRP promotes the biogenesis of a subset of microRNAs. Nature 2009; 459: 1010-1014.

28. Ruggiero T, Trabucchi M, De Santa F, Zupo S, Harfe BD, McManus MT et al. LPS induces $\mathrm{KH}$-type splicing regulatory protein-dependent processing of microRNA-155 precursors in macrophages. FASEB J 2009; 23: 2898-2908.

29. Zhang X, Wan G, Berger FG, He X, Lu X. The ATM kinase induces microRNA biogenesis in the DNA damage response. Mol Cell 2011; 41: 371-383.

30. Gherzi R, Trabucchi M, Ponassi M, Ruggiero T, Corte G, Moroni C et al. The RNA-binding protein KSRP promotes decay of beta-catenin mRNA and is inactivated by PI3K-AKT signaling. PLoS Biol 2006; 5: e5.

31. Serra C, Palacios D, Mozzetta C, Forcales SV, Morantte I, Ripani M et al. Functional interdependence at the chromatin level between the MKK6/p38 and IGF1/PI3K/AKT pathways during muscle differentiation. Mol Cell 2007; 28: 200-213.

32. Brugge J, Hung MC, Mills GB. A new mutational AKTivation in the PI3K pathway. Cancer Cell 2007; 12: 104-107.

33. Rotwein P, Wilson EM. Distinct actions of Akt1 and Akt2 in skeletal muscle differentiation. $J$ Cell Physiol 2009; 219: 503-511.

34. Conejo R, Lorenzo M. Insulin signaling leading to proliferation, survival, and membrane ruffling in C2C12 myoblasts. J Cell Physiol 2001; 187: 96-108.

35. Anderson C, Catoe H, Werner R. MIR-206 regulates connexin43 expression during skeletal muscle development. Nucleic Acids Res 2006; 34: 5863-5871.

36. Lin WJ, Zheng X, Lin CC, Tsao J, Zhu X, Cody JJ et al. Post-transcriptional control of type I interferon genes by KSRP in the innate immune response against viral infection. Mol Cell Biol 2011; 31: 3196-3207.

37. Schiaffino S, Mammucari C. Regulation of skeletal muscle growth by the IGF1-Akt/PKB pathway: insights from genetic models. Skeletal Muscle 2011; 1: 4

38. Yuasa K, Hagiwara Y, Ando M, Nakamura A, Takeda S, Hijikata T. MicroRNA-206 is highly expressed in newly formed muscle fibers: implications regarding potential for muscle regeneration and maturation in muscular dystrophy. Cell Struct Funct 2008; 33: 163-169.

39. Iliopoulos D, Polytarchou C, Hatziapostolou M, Kottakis F, Maroulakou IG, Struhl K et al. MicroRNAs differentially regulated by Akt isoforms control EMT and stem cell renewal in cancer cells. Sci Signal 2009; 2: ra62.

40. Sayed D, Abdellatif M. AKT-ing via microRNA. Cell Cycle 2010; 9: 3213-3217.

41. Michlewski G, Cáceres JF. Antagonistic role of hnRNP A1 and KSRP in the regulation of let-7a biogenesis. Nat Struct Mol Biol 2010; 17: 1011-1018

\section{Supplementary Information accompanies the paper on Cell Death and Differentiation website (http://www.nature.com/cdd)}

